\title{
Free-standing graphene oxide mid-infrared polarizers $\uparrow$
}

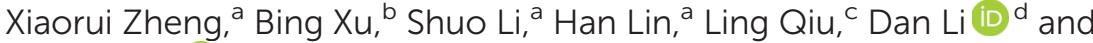 \\ Baohua Jia (iD *a
}

\begin{abstract}
Mid-infrared (MIR) represents a crucial spectral region for applications in spectroscopy, sensing, imaging, security and industry screening, owing to the strong characteristic vibrational transitions of many important molecules. However, the current MIR compatible materials are fragile, hazardous, and costly, which hampers the performance of MIR devices. Here, we developed a versatile transmittance-based KramersKronig method and obtained the optical properties of graphene oxide in the MIR region, unveiling its application potentials as a novel MIR compatible material. As an example, we demonstrated free-standing graphene oxide MIR polarizers with large extinction ratio ( 20 dB) and controllable working wavelength up to $25 \mu \mathrm{m}$, by using the low-cost and flexible direct laser writing technique. Our transmittance-based KK method offers a versatile approach to obtain the optical properties of novel atomic-scale low-dimensional materials in the less developed MIR region and opens up opportunities in high performing functional MIR devices.
\end{abstract}

\section{Introduction}

Mid-infrared (MIR) radiation, particularly in the wavelength range from $3 \mu \mathrm{m}$ to $30 \mu \mathrm{m}$, plays an indispensable role in a wide range of applications, including infrared (IR) imaging and sensing, aerospace engineering, medical science, military defence, and information and communications. ${ }^{1-3}$ However, the MIR (in particular $>8 \mu \mathrm{m}$ ) compatible materials are largely restricted to germanium-, selenide-, and fluoride-based materials such as $\mathrm{Ge}, \mathrm{ZnSe}$ and $\mathrm{BaF}_{2}$, which are fragile, hazardous, and costly. ${ }^{4,5}$ It remains challenging yet crucial to search for appropriate MIR materials, which possess broadband MIR transparency, high refractive index, mechanical and chemical stability, cost-effectiveness and rapid manufacturing capabilities.

The discovery of graphene plasmons has attracted enormous attention due to the potential to reshape the landscape of photonics and optoelectronics in the MIR and terahertz $(\mathrm{THz})$ regimes. $^{6,7}$ However, the considerable plasmon damping, the low cost-effectiveness in fabrication, the poor

${ }^{a}$ Centre for Translational Atomaterials, Swinburne University of Technology, Hawthorn 3122, Australia. E-mail: bjia@swin.edu.au

${ }^{b}$ University of Fribourg, Department of Physics and Fribourg Center for

Nanomaterials, Chemin du Musée 3, CH-1700 Fribourg, Switzerland

${ }^{c}$ Shenzhen Geim Graphene Center, Tsinghua-Berkeley Shenzhen Institute, Tsinghua University, Shenzhen 518055, P. R. China

${ }^{d}$ Department of Chemical Engineering, The University of Melbourne, VIC 3010,

Australia

$\dagger$ Electronic supplementary information (ESI) available. See DOI: 10.1039/ d0nr01619e processability and nanostructuring capability of graphene have placed restrictions for its practical MIR applications. ${ }^{8,9}$ In contrast, the chemically derived graphene oxide (GO) has been seen as an appealing alternative material due to its unique physical and chemical properties arising from the hybridization of the $\mathrm{sp}^{2}$ and $\mathrm{sp}^{3}$ carbon atoms. ${ }^{10}$ Uniquely, the optical and electrical properties of GO have been precisely tailored by manipulating its $\mathrm{sp}^{2}$-hybridized domains during the reduction process, enabling various photonic and optoelectronic applications. ${ }^{11-17}$ However, the current optical characterizations of GO have been limited in the visible or near-infrared (NIR) regimes by using the conventional approaches such as ellipsometry, picometry or microscopy. ${ }^{18,19}$ The optical properties of GO in the MIR regime are less studied previously owing to the constricted selections of both the available MIR characterization approaches and the unrevealed material band models, which hampers its MIR photonic applications. ${ }^{20}$

In this paper, we have demonstrated a model-free KramersKronig (KK) method to characterize the optical properties of GO from the visible ( $200 \mathrm{~nm}$ ) to MIR (up to $25 \mu \mathrm{m}$ ) region by simply measuring its transmission spectrum. Based on the obtained dispersion relations, high performing MIR polarizers on free-standing GO films have been fabricated by using the direct laser writing (DLW) technique, showing a large extinction ratio $(\sim 20 \mathrm{~dB})$ and controllable working wavelengths in MIR region. Both finite-difference time-domain (FDTD) simulation and guided-mode theoretical optimization of the MIR polarizers have been performed and achieve good agreement with the experimental measurements, which further validates 
the extracted MIR dispersion relations of GO by using the KK method. The mechanical robustness, the low-cost manufacturing, and the flexible integration capability of free-standing GO polarizers offer great potential for various photonic applications particularly in the MIR regime. Furthermore, the successful demonstration of the model-free transmittance-based KK method provides a universal approach to access the optical properties of novel low-dimensional materials with the atomiclayer thickness in the less developed MIR or THz regimes.

\section{Results}

\section{Transmittance-based KK method and GO dispersion relation}

The accurate determination of complex refractive index of thin films is crucial from both a fundamental and a technological viewpoint, which requires two independent optical measurements, such as transmittance and reflectance. As a result, it requires sufficiently accurate transmittance and reflectance measurements, giving rise to larger inaccuracies of the extracted complex refractive index. On the contrary, a KK method eliminates the need to combine two types of spectral measurements by calculating the phase shift of the beam from the spectral measurement. In this way, the complex refractive index of the material can be determined from the measured spectrum and the calculated phase shift of the beam. ${ }^{21}$

The experimental flow chart of the transmittance-based KK method has been shown schematically in Fig. 1a. By combining the spectrometers in different frequency ranges, a broadband light radiation (from ultraviolet (UV) to MIR in our case) is incident on a free-standing GO thin film, and the transmittance $\left(T_{\mathrm{f}}\right)$ has been acquired experimentally. Then the corresponding phase shift $(\psi)$ of the transmitted radiation has been determined through a transmittance-based KK transform: ${ }^{22}$

$$
\psi(\omega)=\frac{2 \omega}{\pi} P \int_{0}^{\infty} \frac{\ln \left[T_{\mathrm{f}}\left(\omega^{\prime}\right)\right]^{1 / 2}}{\omega^{\prime 2}-\omega^{2}} \mathrm{~d} \omega^{\prime}-2 \pi \omega d,
$$

where $\omega$ is the frequency of the incident wave and $d$ is the film thickness. The $P$ before the integral indicates that the Cauchy principle value of the integral should be used. ${ }^{23}$ Given the correlated transmittance $\left(T_{\mathrm{f}}\right)$ and phase shift $(\psi)$, the dispersion relations of both the refractive index $(n)$ and the extinction coefficient $(\kappa)$ have be calculated through a model-free inverse relation: ${ }^{22}$

$$
\left\{\begin{array}{c}
T_{\mathrm{f}}=16\left(n^{2}+\kappa^{2}\right) /\left(C^{2}+D^{2}\right) \\
\psi=\arctan (\kappa C+n D) /(\kappa D-n C)
\end{array}\right.
$$

where

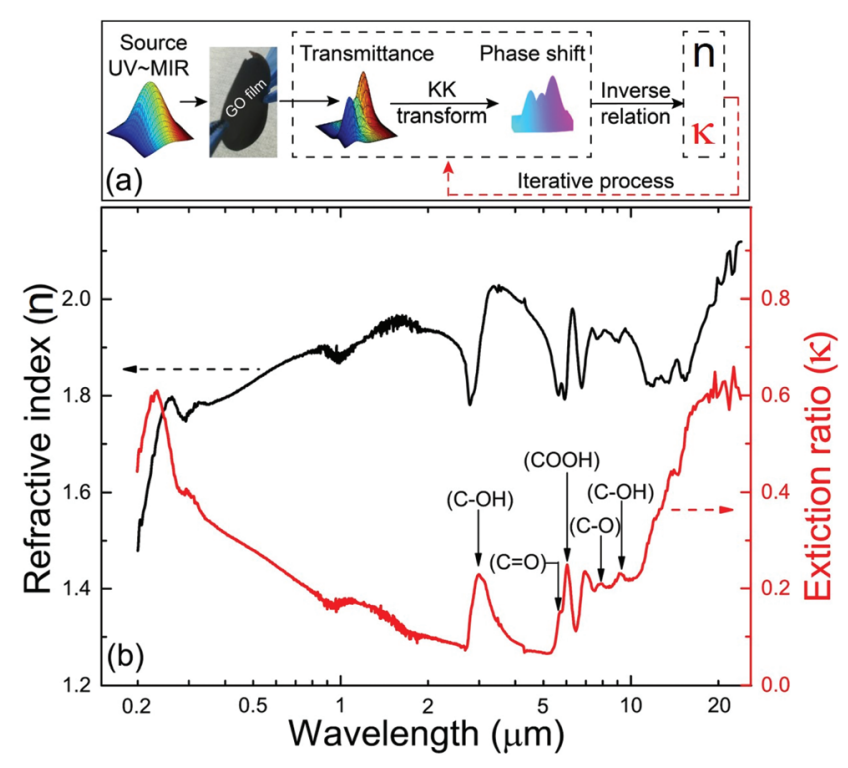

Fig. 1 Complex refractive index of graphene oxide in the MIR regime. (a) The experimental flow chart of the model-free transmittance-based KK method. (b) The dispersion relations of a free-standing GO film ( $30 \mathrm{~nm}$ thick).

and $n_{\mathrm{s}}$ is the refractive index of the substrate $\left(n_{\mathrm{s}}=1\right.$ in our case). A computer-assisted curve fitting program has been developed to calculate $n$ and $\kappa$ values from $T_{\mathrm{f}}$ and $\psi$. Finally, an iterative process has been applied to further improve the accuracy of $n$ and $\kappa$ values. Compared with the polarization sensitive optical characterization methods such as ellipsometry, ${ }^{19}$ our transmittance-based KK method has be performed at the normal incidence, which largely reduces the influences of the roughness and contamination of the sample surface. Moreover, no assumption of the physical model or the material band shape is required as in the case of ellipsometry, ${ }^{19}$ which makes it an ideal approach especially for the newly developed materials. Furthermore, the attainable bandwidth of the dispersion relations is solely determined by the measurable spectrum range. ${ }^{22}$ As a result, the full spectrum range of the dispersion relation is accessible by combining various spectroscopic methods, offering the critical optical properties in the less developed MIR or THz range. The detailed experiment and calculation procedures of the transmittance-based KK method are given in Part I of ESI. $\dagger$

The thinner of the film, the higher accuracy of transmittance-based KK method. The GO film thickness should be less

$$
\left\{\begin{array}{l}
C=e^{K}\left\{\left[(1+n)\left(n+n_{s}\right)-\kappa^{2}\right] \cos N+\kappa\left(1+2 n+n_{s}\right) \sin N\right\}+e^{-K}\left\{\left[(1-n)\left(n-n_{s}\right)+\kappa^{2}\right] \cos N-\kappa\left(1-2 n+n_{s}\right) \sin N\right\} \\
D=e^{K}\left\{\left[(1+n)\left(n+n_{s}\right)-\kappa^{2}\right] \sin N-\kappa\left(1+2 n+n_{s}\right) \cos N\right\}-e^{-K}\left\{\left[(1-n)\left(n-n_{s}\right)+\kappa^{2}\right] \sin N+\kappa\left(1-2 n+n_{s}\right) \cos N\right\}
\end{array}\right.
$$

in which

$$
\left\{\begin{array}{l}
K=2 \pi \kappa d / \lambda \\
N=2 \pi n d / \lambda
\end{array}\right.
$$

than one tenth of the measuring wavelength, which in our case is from $0.2 \mu \mathrm{m}-25 \mu \mathrm{m}$. Meanwhile, to ensure the accuracy of the measured transmittance, the elimination of substrate is desired, but challenging in particular for ultrathin GO films. Moreover, the minimal beam size of the spectrometer light 
source is in centimeter scale. Hence, a centimeter size and ultrathin $(<50 \mathrm{~nm})$ GO film has to be made free-standing and robust for measurements using various spectroscopy facilities. Owing to our unique GO film synthesis technique, the flexible transferring capability and the mechanical strength, ${ }^{11,12}$ we have successfully prepared a $30 \mathrm{~nm}$ thick freestanding GO film and accurately obtained its transmittance over the large wavelength range from $0.2-25 \mu \mathrm{m}$ without any damages.

The dispersion relations of a free-standing GO film ( $\sim 30 \mathrm{~nm}$ thick) characterized by using the transmittance-based KK method have been shown in Fig. 1b. It is evident that both the $n$ and $\kappa$ have been successfully extended to $25 \mu \mathrm{m}$, unveiling the abundant optical characteristics of GO from UV to MIR region. In addition, we have performed ellipsometric measurements of a GO film in the ultraviolet-visible (UV-Vis) region (see Fig. S1 in ESI $\dagger$ ). A good agreement between the ellisomeric method and KK method has been achieved, which further proves the accuracy of the model-free KK method. As in the case of doped graphene, ${ }^{24}$ Pauli-blocking of the strong interband optical transitions in the $\mathrm{sp}^{2}$ domains of GO has been validated from the small $\kappa$ value between $2 \mu \mathrm{m}$ to $5 \mu \mathrm{m}$, indicating the shift of the Fermi level in GO due to the hybridization of the $\mathrm{sp}^{2}$ and $\mathrm{sp}^{3}$ domains. ${ }^{25}$ Moreover, the characteristic oxygen configurations in the GO structure, including hydroxyl $(\mathrm{C}-\mathrm{OH})$, ketonic species $(\mathrm{C}=\mathrm{O})$, carboxyl $(\mathrm{COOH})$ and epoxide (C-O), have been clearly identified and labelled on the $\kappa$ curve, which is consistent with the previous Fourier-transform infrared (FTIR) spectrum of GO. ${ }^{26,27}$ In addition, a Drude peak response at the wavelength $>10 \mu \mathrm{m}$ has been observed owing to the increase of $\kappa$, which is due to the intraband free carriers absorption. ${ }^{7}$ An overall examination of $\kappa$ shows a low level $(\kappa<0.2)$ of absorption in the wavelength range $<10 \mu \mathrm{m}$ and an acceptable absorption $(0.2<\kappa<0.6)$ up to $25 \mu \mathrm{m}$, which is promising given its ultrathin thicknesses. For example, a transmittance around $90 \%$ for a GO film with the thickness up to $300 \mathrm{~nm}$ and a transmittance around $80 \%$ for a GO film with the thickness up to $600 \mathrm{~nm}$ have been illustrated, indicating the usability of GO film with reasonable thickness in the longwave infrared (LWIR) regime (see Fig. S2 in ESI $\dagger$ ). Meanwhile, a comparably large $n(\sim 2)$ has been observed, further confirming the ultra-broadband dispersion relations of GO from the visible to the MIR regions. Therefore, the measured dispersion relations of GO demonstrate the accuracy of the transmittance-based KK method, and more importantly, provide the essential optical parameters for the realization of various photonic devices especially in the MIR region. It should be noted that the extracted index at two spectrum edges, which are $0.2 \mu \mathrm{m}$ and $25 \mu \mathrm{m}$, cannot be validated due to the truncated spectrum used in the KK method. All our simulations are performed based on the extracted index other than these two wavelength points.

\section{Design and manufacturing of MIR GO polarizers}

The extracted dispersion relation of GO offers promising potentials for realizing functional MIR devices. Compared with the largely developed MIR sources and detectors, ${ }^{3,28,29}$ it is still challenging to realize an efficient MIR polarizer that has mechanical robustness, non-toxic constitute materials, costeffective manufacturability and the high performance over the entire MIR range, especially beyond the silicon transparency window $(>8 \mu \mathrm{m}) .^{30-32}$ To address these challenges, we have proposed a MIR polarizer formed by the periodic GO ribbons, as shown schematically in Fig. 2a. Owing to the superb mechanical strength of the GO film, free-standing GO polarizer is constructed to completely eliminate the absorptions of the supporting substrates, which is yet challenging for the current MIR polarizers. ${ }^{30-33}$ As a result, the operational wavelengths of the polarizers, which have previously been restricted by the selection of the supporting substrate, are extended to LWIR regime by using our free-standing GO polarizers. The DLW technique has been demonstrated previously to pattern arbitrary structures on GO films as a one-step mask-free process. ${ }^{12,15}$ As a result, free-standing GO films with grating structures have been realized by using our DLW setup (see Methods and Fig. S3 in ESI $\dagger$ ). In comparison with cleanroom semiconductor nanofabrication methods such as photo lithography, nano imprinting or electron beam lithography, which
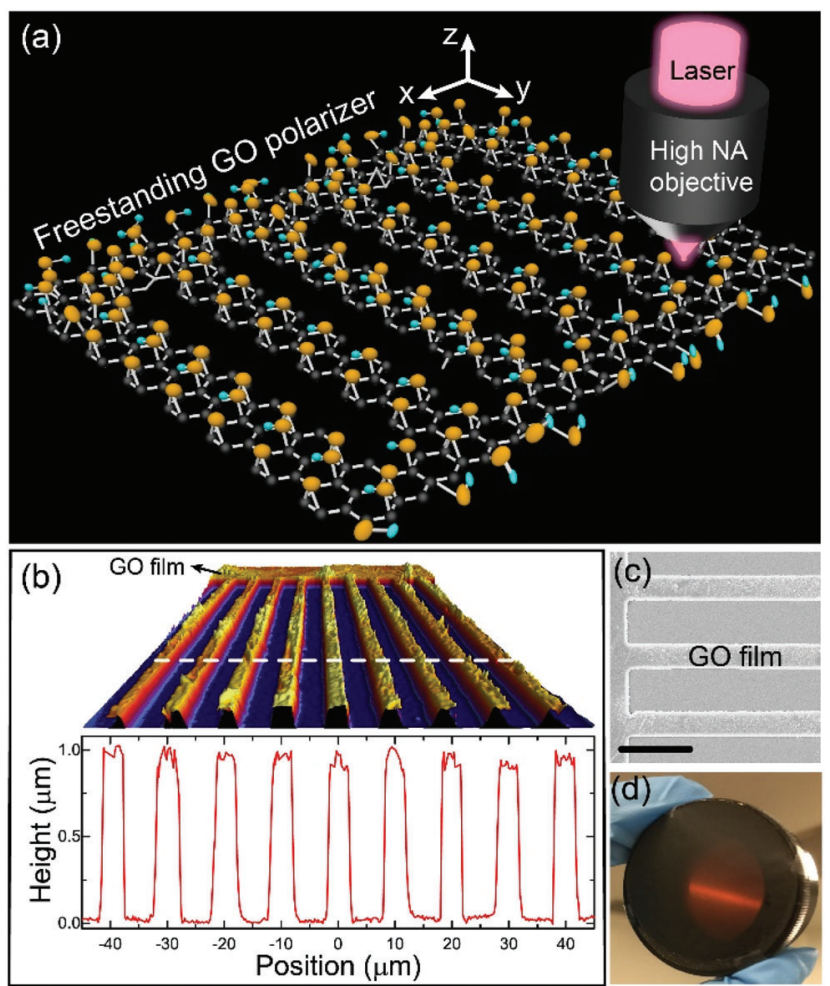

Fig. 2 Free-standing graphene oxide MIR polarizers. (a) Conceptual design and laser manufacturing of the free-standing GO polarizer. (b) Top: The 3D topographic view of the GO polarizer measured with an optical profiler. Bottom: The cross-sectional surface profile of the GO polarizer along the white dashed line marked in the top 3D topographic view. (c) The SEM image of the fabricated GO polarizer. Scale bar: $10 \mu \mathrm{m}$. (d) The photo of a free-standing GO polarizer mounted on a commercially available standard polarizer mount. 
are generally used for fabricating existing IR devices, ${ }^{31-34}$ the laser manufacturing capability of the GO films offers a lowcost, time-efficient, mass-producible and flexible fabrication solution towards the industrial and commercial applications of our GO polarizers.

The surface morphology of a GO polarizer fabricated on a $1 \mu \mathrm{m}$-thick GO film has been characterized by using the $3 \mathrm{D}$ optical profiler (see Methods), as shown in Fig. 2b (top). The cross-sectional surface profile along the white dashed line is also plotted in Fig. 2b (bottom), showing the well-defined periodic GO ribbons with the periodicity of $10 \mu \mathrm{m}$ and a ribbon width of $4 \mu \mathrm{m}$. Moreover, the surface roughness of the GO ribbons has been controlled below $10 \%(<100 \mathrm{~nm}$ in this case) of the overall GO film thickness $(\sim 1 \mu \mathrm{m})$, which improves the performance of the GO polarizer by reducing its interactions with the MIR radiations including the unwanted diffraction or scattering. The scanning electron microscope (SEM) is also used to illustrate the high quality of the laser fabricated GO polarizer, as shown in Fig. 2c. The sharp edges of the GO ribbons have been achieved by using the high resolution DLW technique. In addition, our free-standing GO thin film polarizer is readily integrated with the commercial polarizer mounts (Fig. 2d) owing to the mechanical strength and the flexibility of the GO film, offering the promising potentials towards integrated devices.

\section{Working principle of the GO polarizer}

The working principle of the proposed GO polarizer has been shown schematically in Fig. 3a. The free-standing GO polarizer is made of a periodic dielectric waveguide array dispensed along the $x$-axis, forming by separated high index GO ribbons stretched along the $y$ direction. The geometrical configuration of the GO polarizer is defined by the GO film thickness $(d)$, the periodicity of the GO ribbons $(a)$ and the width of the GO ribbon $(l)$, as labelled in Fig. 3a. Upon illumination by an unpolarized light beam, the TE polarization (red arrows) with the electric field parallel to the high index GO ribbons is coupled into the GO film via the guided-mode resonances, ${ }^{35-38}$ propagating in the plane (see Fig. 3a). In comparison, the TM polarization (blue arrows) with the electric field perpendicular to the GO ribbons is allowed to pass through. As a result, the proposed periodic GO ribbons split the two orthogonally orientated polarizations, becoming an effective TM polarizer.

To further investigate the coupling mechanisms of the TE polarization, the electric field distributions within the cross section of GO ribbons are calculated numerically for a GO polarizer with $d=1 \mu \mathrm{m}, a=4 \mu \mathrm{m}, l=2 \mu \mathrm{m}$, which are represented by one exemplary ribbon (white dashed square in Fig. 3a). Two coupling modes have be identified as the guidedmode with the electric field resonance in the $x-y$ plane and the waveguide mode with the electric field resonance along the $z$ direction, which gives rise to the strong polarization sensitivity of GO polarizers (see Part II in ESI $\dagger$ ). The performance of the proposed GO polarizer has then been studied by using the

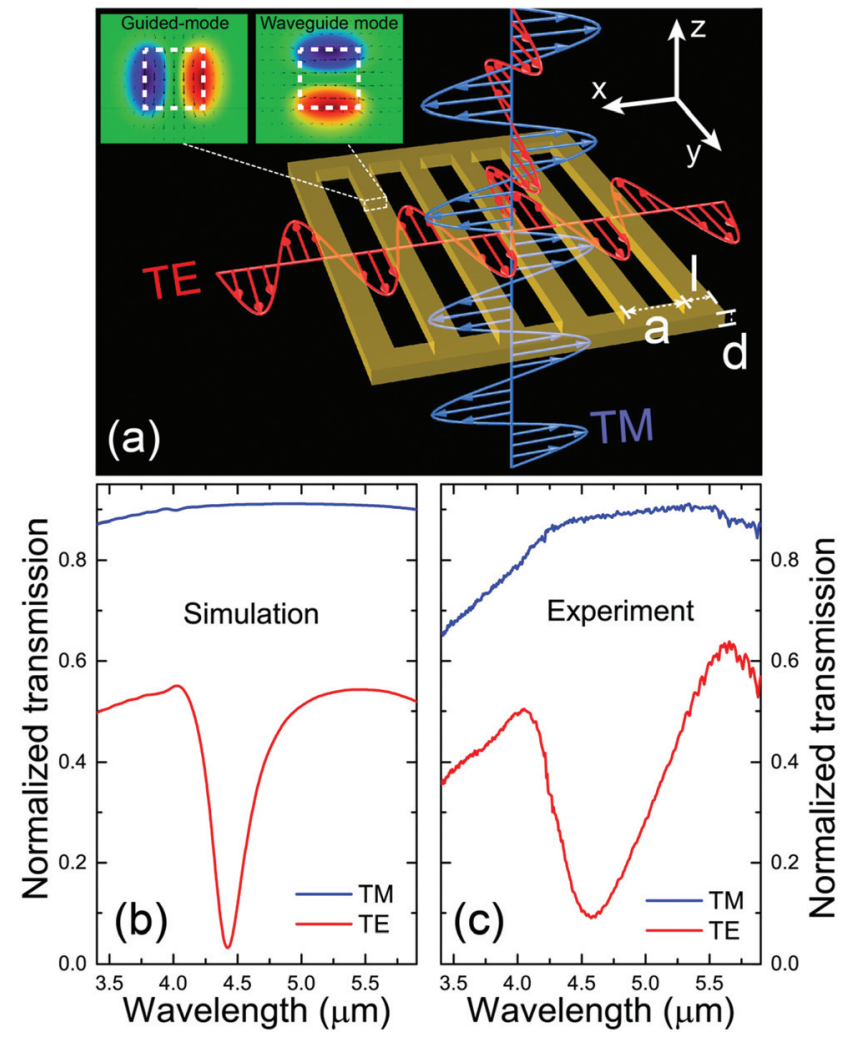

Fig. 3 The operations of graphene oxide MIR polarizers. (a) Schematic figure of the working principle of the GO polarizer. Insets: The crosssectional $(x-z$ plane) electric field distributions within one exemplary GO rod (white dashed line) have been plotted for both guided-mode (left) and waveguide mode (right) respectively. (b) The FDTD simulation of the transmission spectra of the proposed GO polarizer under both TM and TE polarizations. (c) The experimental transmission spectra of the proposed GO polarizer measured by using the microscope FTIR spectrometer.

numerical FDTD simulations (see Methods). The MIR transmission spectra of the GO polarizer $(d=1 \mu \mathrm{m}, a=4 \mu \mathrm{m}, l=$ $2 \mu \mathrm{m}$ ) under both the TE and TM polarizations have been simulated by using the measured GO dispersion relations in the MIR regime, as shown in Fig. 3b. The transmittance as large as $90 \%$ has been observed for the TM polarized light, whereas the TE polarized light is largely blocked with a minimal transmittance of $<3 \%$ at the wavelength of around $4.5 \mu \mathrm{m}$, behaving as a high performance TM polarizer in the MIR regime. Furthermore, we have simulated the reflection and absorption spectra of the GO polarizer under TE polarization, showing prominent reflection and absorption of the TE light (see Fig. S4 in ESI $\dagger$ ). We propose that the TE light couples into the GO gratings and propagates within the GO film (see Fig. 3a), which results in the partial absorption of the TE light. Meanwhile, TE light is continually decoupled out of the waveguide, and interferes with both the transmitted and reflected waves. At resonance, the decoupled light interferes destructively with the transmitted light, and constructively 
with the reflected light, leading to efficient resonant reflection. ${ }^{37,38}$

The free-standing GO polarizer with the same geometry as the simulation has then been fabricated experimentally by using the DLW technique (see Methods and Fig. S3 in ESI $\dagger$ ). The performance of the fabricated free-standing GO polarizer with an overall size of $100 \times 100 \mu \mathrm{m}^{2}$ has be characterized by using a microscope FTIR spectrometer (see Methods). A pin hole system has been designed to reduce the incident angle variation $\left(\sim 5^{\circ}\right)$ and ensure the normal incidence condition (see Fig. S5 in ESI $\dagger$ ). A larger pin hole gives rise to a better signal to noise ratio, but increases the incident angle variations, which results in broadening of the resonances in the spectrum. ${ }^{39}$ Therefore, we have specifically used a smaller pin hole (incident angle variation $\sim 5^{\circ}$ ) to ensure the normal incidence condition is satisfied to eliminate the broadening of the spectrum and study the operation mechanisms of the polarizers. Fig. 3c shows the measured transmission spectra of the free-standing GO polarizer under TE and TM polarizations, respectively. The splitting of the two polarization states has been observed at around $4.5 \mu \mathrm{m}$, indicating a good agreement with the FDTD simulation. The slight discrepancies, e.g. broadening of the TE resonance, are due to the non-normal light incidence during the microscope FTIR measurements. Furthermore, we have simulated the influence of the incident angle and observed the broadening of the transmission dips under TE polarization (see Fig. S6 in ESI†). To this end, the large polarization sensitivity of the free-standing GO polarizer has been demonstrated both numerically and experimentally, indicating a promising linear MIR polarizer with high efficiency and performance. Moreover, the good agreement between the FDTD simulation and the measurements validates the high accuracy of the transmittance-based KK method in extracting dispersion relation of GO in the MIR regime.

\section{Broadband tunability}

The working wavelength of commercial MIR polarizers is restricted by the transparent window of the supporting substrates. A wire-grid polarizer on $\mathrm{Y}_{2} \mathrm{O}_{3}$ substrate with the practical useful efficiency $(>70 \%)$ has the wavelength limit up to $10 \mu \mathrm{m},{ }^{32}$ whereas the grating polarizer on $\mathrm{MgF}_{2}$ substrate have the limit up to $3.4 \mu \mathrm{m} .{ }^{40}$ Recently, a sulfuric polymer poly (sulfur-random-(1,3-diisopropenylbenzene)) material has been developed with a low loss in the IR range up to $6 \mu \mathrm{m} .{ }^{41}$ Although a silver-film polarizers have been demonstrated in the IR range between $10 \mu \mathrm{m}$ to $25 \mu \mathrm{m},{ }^{33}$ a double-sided subwavelength grating structure needs to be patterned first in silicon and then coated by silver-film, resulting in nanofabrication challenges as well as high cost. In comparison, the working wavelength of our GO polarizer has been designed over a broadband MIR wavelength range owing to the free-standing configuration, the broadband dispersion relations of GO, the scaling law of the guided-mode coupling, and the flexible patterning capability using DLW method. In particular, the working wavelength of our GO polarizers is determined by the periodicity (a) according to the phase matching condition for the guided-mode coupling: ${ }^{35,37}$

$$
\lambda_{\mathrm{g}}=a \cdot\left(n_{\mathrm{eff}}-n_{\mathrm{c}} \sin \theta\right),
$$

where $\lambda_{\mathrm{g}}$ is the wavelength of the guided-mode resonance, $n_{\mathrm{eff}}$ is the effective refractive index of the guided-mode, $n_{\mathrm{c}}(=1)$ is the refractive index of air and $\theta$ is the incident angle $\left(=0^{\circ}\right.$ for the normal incidence). Therefore, we have fabricated GO polarizers with varying periodicities from $2 \mu \mathrm{m}$ to $12 \mu \mathrm{m}$ on the same GO film (1 $\mu \mathrm{m}$ thick) to tune their working wavelength in a range from $2 \mu \mathrm{m}$ to $14 \mu \mathrm{m}$ (the experimental limit of the microscope FTIR spectrometer). The microscope images of the fabricated GO polarizers have been shown in the insets of Fig. 4b. Uniform free-standing GO ribbons with sharp edges have been patterned for various periodicities from $2 \mu \mathrm{m}$ to $12 \mu \mathrm{m}$ by using DLW technique. As shown in Fig. 4a, the transmission spectra of eight free-standing GO polarizers under TE (solid curves) and TM (dashed curves) polarizations have been measured by FTIR. The TE transmission minima have been observed for all the GO polarizers, and the positions are found to redshift as the increase of the periodicity, obeying the scaling law of the guided-mode resonance. However, the minimal transmittance increases for larger periodicities, which deteriorates the extinction ratio of the GO polarizers.

The reason for the performance deterioration is attributed to the less overlapping between guided-mode resonance and waveguide mode resonance. The guided-mode resonance in the $x-y$ plane is correlated with the grating periodicity $(a)$, whereas the waveguide mode resonance along the $z$ direction is determined by the structural confinement in the $z$ direction, which is the GO film thickness $(d)$. As a result, the overlapping of the two coupling mechanisms has been altered for the different periodic GO gratings fabricated on the same GO film. This has been evidently illustrated from the broadening of the transmission spectra for the larger periodicities $(a>4 \mu \mathrm{m})$. The transmission spectra under the TM polarization indicate that the considerable polarization sensitivity is maintained for all GO polarizers. Furthermore, a large transmission efficiency $(\sim 90 \%)$ of the desirable polarization has be achieved from $4 \mu \mathrm{m}$ to $14 \mu \mathrm{m}$ (the equipment limit), offering potentials for highly efficient linear polarizers in LWIR regime.

The dependence of the working wavelength as a function of the periodicity has been further investigated by using the numerical FDTD simulations and the theoretical calculations. Firstly, the working wavelength has been extended up to $25 \mu \mathrm{m}$ (limit of the measured dispersion relations) according to the FDTD simulations, as shown in Fig. 4b (black dashed line). Moreover, the theoretical prediction of the working wavelength following the scaling law (eqn (1)) has also been provided based on the measured dispersion relations, as shown in Fig. 4b (red solid line). As a reference, the experimental working wavelength of the GO polarizers has been plotted as a function of the periodicity, as shown in Fig. 4b (black spheres). Good consistence has been achieved among the experiments, the FDTD simulations and the theoretical calcu- 

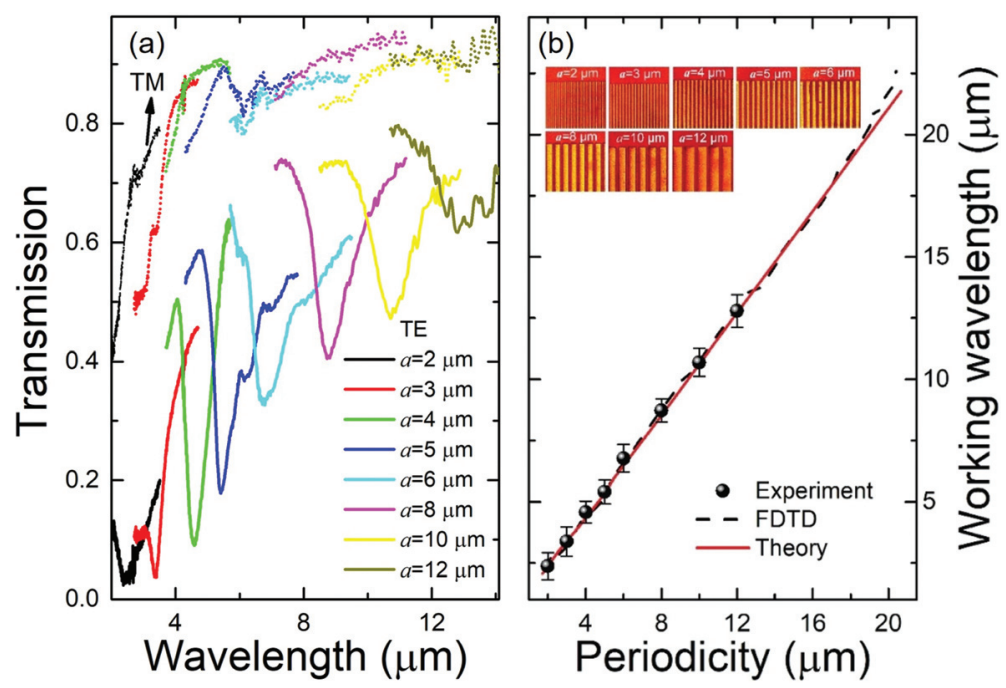

Fig. 4 Broadband tunability of graphene oxide MIR polarizers. (a) Experimental broadband tuning of the working wavelengths of GO polarizers from $2 \mu \mathrm{m}$ to $14 \mu \mathrm{m}$. (b). The experimental dependence of the working wavelength as a function of the periodicity of the GO polarizer comparing to the FDTD simulations and the theoretical calculations. Inset: The microscope images of the fabricated GO polarizers with varying periodicities.

lations, which further validates the accuracy of the dispersion relation measured by the transmittance-based KK method. As a result, additional functional GO devices in the MIR regime can be conveniently designed based on its dispersion relation, opening up new avenues for the challenging MIR applications. It should also be noted that the dispersion relation of GO is readily extended beyond $25 \mu \mathrm{m}$ (the experimental limit of our Bench FTIR spectrometer) to the far-infrared or THz regime by utilizing the transmittance-based KK method.

\section{Optimization of extinction ratio}

The performance of a linear polarizer is characterized by the extinction ratio, defined as the ratio of the power in the desired polarization to the power in the undesired polarization. To investigate the performance of our GO polarizers, we have simulated the extinction ratio as a function of both the GO film thickness $(d)$ and the periodicity $(a)$ by using the FDTD simulations, as shown in Fig. 5a. The width of the GO ribbon $(l)$ is fixed at $0.2 a$, maintaining the same filling ratio of the periodic waveguide array. The extinction ratio of GO polarizers is found to be highly related to the GO film thickness and the grating periodicity (see Fig. 5a). The white circles in Fig. 5a represent the geometrical configurations of the fabricated GO polarizers (insets of Fig. 4b) with the increasing periodicity on the same $1 \mu \mathrm{m}$-thick GO film. As the periodicity increases from $2 \mu \mathrm{m}$ to $8 \mu \mathrm{m}$, a degradation of the extinction ratio is identi-

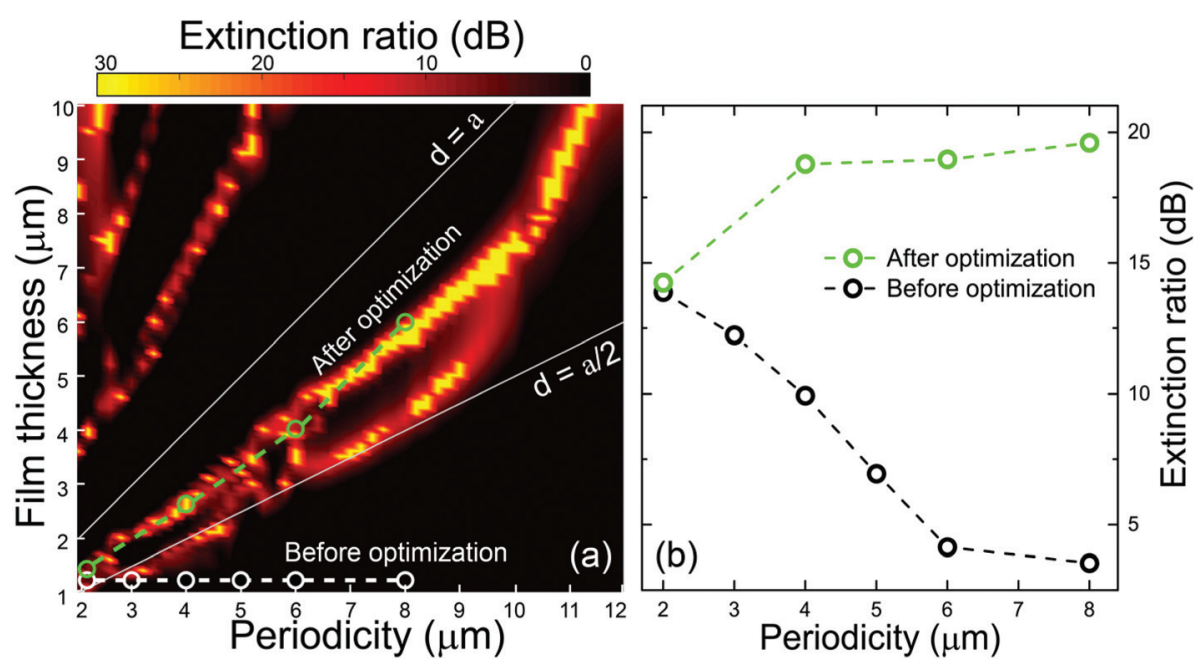

Fig. 5 Optimizations of graphene oxide MIR polarizers. (a) The FDTD simulations of the extinction ratio as the function of both the GO film thickness and the grating periodicity. The theoretical prediction of the optimized design $(a / 2<d<a)$ has been indicated by the white solid lines. The white and green circles represent the geometries of the fabricated GO polarizers before and after the optimization respectively. (b) Experimental results of the extinction ratio before and after the optimization. 
fied from the FDTD simulations. After examining the measured transmission spectra of the corresponding GO polarizers (Fig. 4a), the extinction ratio has been plotted in Fig. 5b (black circles). The extinction ratio of the GO polarizer reaches as large as $14 \mathrm{~dB}$ with a periodicity of $2 \mu \mathrm{m}$, and reduces to less than $3 \mathrm{~dB}$ as the increase of the periodicity (up to $8 \mu \mathrm{m}$ ). As a result, good consistence of the extinction ratio between the FDTD simulation and the experimental results has been achieved.

To optimize the extinction ratio of GO polarizers, the overlapping between guided-mode resonance and waveguide mode resonance has been investigated. It is obvious that both of the two coupling mechanisms will block the incident radiations and increase extinction ratio. Therefore, the optimized extinction ratio will be achieved when both the guided-mode and the waveguide mode are excited at the same wavelength. For the waveguide mode coupling along the $z$ direction, the cut-off wavelength $\lambda_{\mathrm{c}}$ has been expressed as (see also Part II in ESI $\dagger$ ): ${ }^{42}$

$$
\lambda_{\mathrm{c}}=n_{\mathrm{GO}} \cdot d,
$$

where $n_{\mathrm{GO}}$ is the refractive index of the GO film. As a result, based on eqn (1) and (2), the overlapping of the two coupling modes $\left(\lambda_{\mathrm{c}}=\lambda_{\mathrm{g}}\right)$ requires:

$$
n_{\mathrm{GO}} \cdot d=a \cdot n_{\mathrm{eff}} .
$$

Given the fact that $1<n_{\mathrm{eff}}<n_{\mathrm{GO}}<2$ the GO film thickness (d) should satisfy $a / 2<d<a$ to ensure the mode overlapping for the higher extinction ratio (marked with white lines in Fig. 5a) which shows good agreement with the FDTD simulations.

Based on the optimized extinction ratio regions in Fig. 5a three GO polarizers have been fabricated with the carefully designed periodicities and the GO film thicknesses marked as the green circles in Fig. 5a. The transmission spectra of the three optimized GO polarizers have been characterized by using the microscope FTIR spectrometer (see Fig. S7 in ESI $\dagger$ ) and the extinction ratio are extracted as shown in Fig. 5b (green circles). As a result the optimized extinction ratio as large as $20 \mathrm{~dB}$ has been realized experimentally in the MIR range which is comparable with the commercialized holographic wire-grid polarizers (cost over $\$ 2000) .{ }^{43}$ In contrast the cost of our GO polarizers is estimated to be low in consideration of the low-cost DLW method and the abundance of GO materials.

\section{Conclusion}

We have unveiled experimentally the dispersion relation of GO by using the model-free transmittance-based KK method providing the fundamental optical parameters of GO for various photonic and optoelectronic applications in the MIR regime. As an example we realized the free-standing MIR GO polarizers with controllable working wavelengths from $2 \mu \mathrm{m}$ to $14 \mu \mathrm{m}$ large extinction ratio of $20 \mathrm{~dB}$ flexible integration capability and low-cost manufacturing. Two coupling mechanisms of the
GO polarizers have been analysed and good agreement has been reached between the theoretical and experimental results.

The development of the model-free transmittance-based KK method in characterizing the ultrathin GO films opens up a promising approach to access the optical properties of novel low-dimensional materials with atomic-scale thicknesses which is still challenging by using the conventional spectroscope ellipsometry method. Moreover the attainable frequency range is extendable by combining various spectroscopic methods from the UV to microwave ranges promoting the design of functional integrated photonic devices in the less developed MIR and THz regime.

\section{Methods}

\section{Direct laser writing}

A homemade DLW system was utilized to fabricate the freestanding GO polarizers by using a low repetition rate femtosecond pulsed laser beam (100 fs pulse $10 \mathrm{kHz} 800 \mathrm{~nm}$ ). The schematic figure showing the laser fabrication process of the free-standing polarizers is shown as Fig. S3 in ESI. $\dagger$ First we have prepared a glass slide with a round hole in the centre. Then we transferred the GO film onto the glass slide and covered the centric hole yielding a free-standing area of the GO film above the centric hole. Then we covered the GO film with a standard cover slip with the thickness of $0.17 \mathrm{~mm}$. A laser beam was focused through a high numerical aperture (NA = 0.95) objective to fabricate the grating structures in the GO film. Finally after the laser fabrication the cover slip was removed carefully without peeling off the GO film. Therefore the free-standing GO film with the patterned grating area has been achieved and characterized using FTIR.

\section{Surface morphology characterization}

The 3D optical profiler (Bruker ContourGT InMotion) have been used to characterize the surface morphology of the fabricated GO polarizers.

\section{FDTD simulation}

The FDTD simulation was performed by using Lumerical FDTD software. In the simulation the parameters of the GO materials were obtained by experimentally measured dispersion relations. The GO polarizer is defined by a unit cell consisting of one grating pattern and the periodic boundary condition. The plane wave light source has been placed in a normal incident direction (along the $Z$ axis in Fig. 3) on top of the GO polarizer at a distance of $20 \mu \mathrm{m}$ away. TE and TM polarizations of the incident plane wave have been defined according to Fig. 3. The transmission monitor has been placed at the bottom with a distance of $20 \mu \mathrm{m}$ away from the GO polarizer in the normal incident direction. 


\section{Spectroscopic methods}

The UV-Vis transmission spectrum of the GO thin film is measured by using the PerkinElmer UV/Vis Spectrophotometer from $200 \mathrm{~nm}$ to $2 \mu \mathrm{m}$. The IR transmission spectrum of the GO thin film is measured by using the bench FTIR spectrometer (Bruker V70) from $2 \mu \mathrm{m}$ to $25 \mu \mathrm{m}$. The IR transmission spectrum of the GO polarizer with the limited sample size is measured by using the microscope FTIR spectrometer (Bruker V70 and Hyperion 2000) from $2 \mu \mathrm{m}$ to $14 \mu \mathrm{m}$. The microscope FTIR spectrometer uses an FTIR condenser to focus the broadband IR radiations onto the sample in the free space. The normal incidence condition has been achieved by using a pin hole system (see Fig. S5 in ESI $\dagger$ ).

\section{Conflicts of interest}

There are no conflicts of interest to declare.

\section{Acknowledgements}

B. J. acknowledges support from the Australia Research Council through the Discovery Project scheme (Grant No. DP190103186) and Industrial Transformation Training Centres scheme (Grant No. IC180100005). All authors acknowledge the support from the Advanced Solar Facility at Swinburne University of Technology.

\section{References}

1 D. Rodrigo, et al., Mid-infrared plasmonic biosensing with graphene, Science, 2015, 349, 165-168, DOI: 10.1126/ science.aab2051.

2 S. S. Kim, C. Young and B. Mizaikoff, Miniaturized midinfrared sensor technologies, Anal. Bioanal. Chem., 2008, 390, 231-237, DOI: 10.1007/s00216-007-1673-5.

3 P. Werle, et al., Near- and mid-infrared laser-optical sensors for gas analysis, Opt. Lasers Eng., 2002, 37, 101-114, DOI: 10.1016/s0143-8166(01)00092-6.

4 R. Soref, Mid-infrared photonics in silicon and germanium, Nat. Photonics, 2010, 4, 495-497, DOI: 10.1038/ nphoton.2010.171.

5 D. C. Harris, Durable 3-5 mu m transmitting infrared window materials, Infrared Phys. Technol., 1998, 39, 185201, DOI: 10.1016/s1350-4495(98)00006-1.

6 A. N. Grigorenko, M. Polini and K. S. Novoselov, Graphene plasmonics, Nat. Photonics, 2012, 6, 749-758, DOI: 10.1038/ nphoton.2012.262.

7 T. Low and P. Avouris, Graphene Plasmonics for Terahertz to Mid-Infrared Applications, ACS Nano, 2014, 8, 10861101, DOI: 10.1021/nn406627u.

8 H. G. Yan, et al., Damping pathways of mid-infrared plasmons in graphene nanostructures, Nat. Photonics, 2013, 7, 394-399, DOI: 10.1038/nphoton.2013.57.
9 Y. Zhou and K. P. Loh, Making Patterns on Graphene, Adv. Mater., 2010, 22, 3615-3620, DOI: 10.1002/adma.201000436.

10 K. P. Loh, Q. L. Bao, G. Eda and M. Chhowalla, Graphene oxide as a chemically tunable platform for optical applications, Nat. Chem., 2010, 2, 1015-1024, DOI: 10.1038/ nchem.907.

11 X. Zheng, B. Jia, X. Chen and M. Gu, In Situ Third-Order Non-linear Responses During Laser Reduction of Graphene Oxide Thin Films Towards On-Chip Non-linear Photonic Devices, Adv. Mater., 2014, 26, 2699-2703, DOI: 10.1002/ adma.201304681.

12 X. R. Zheng, et al., Highly efficient and ultra-broadband graphene oxide ultrathin lenses with three-dimensional subwavelength focusing, Nat. Commun., 2015, 6, 8433, DOI: 10.1038/ncomms9433.

13 X. R. Zheng, H. Lin, T. S. Yang and B. H. Jia, Laser trimming of graphene oxide for functional photonic applications, J. Phys. D: Appl. Phys., 2017, 50, 074003, DOI: 10.1088/1361-6463/aa54e9.

14 T. S. Yang, H. Lin, X. R. Zheng, K. P. Loh and B. H. Jia, Tailoring pores in graphene-based materials: from generation to applications, J. Mater. Chem. A, 2017, 5, 1653716558, DOI: 10.1039/c7ta04692h.

15 H. Lin, et al., A 90-nm-thick graphene metamaterial for strong and extremely broadband absorption of unpolarized light, Nat. Photonics, 2019, 13, 270-276, DOI: 10.1038/ s41566-019-0389-3-+.

16 H. L. Keng-Te Lin, Y. Tieshan and J. Baohua, Structured graphene metamaterial selective absorbers for high efficiency and omnidirectional solar thermal energy conversion, Nat. Commun., 2020, 11, 1389.

17 Y. Y. Yang, et al., Graphene-Based Multilayered Metamaterials with Phototunable Architecture for on-Chip Photonic Devices, ACS Photonics, 2019, 6, 1033-1040, DOI: 10.1021/acsphotonics.9b00060.

18 I. Jung, et al., Simple approach for high-contrast optical imaging and characterization of graphene-based sheets, Nano Lett., 2007, 7, 3569-3575, DOI: 10.1021/nl0714177.

19 I. Jung, et al., Characterization of thermally reduced graphene oxide by imaging ellipsometry, J. Phys. Chem. C, 2008, 112, 8499-8506, DOI: 10.1021/jp802173m.

20 D. Ma, X. Li, Y. Guo and Y. Zeng, Study on IR properties of reduced graphene oxide, IOP Conf. Ser.: Earth Environ. Sci., 2018, 108, 022019.

21 P. O. Nilsson, Determination of Optical Constants from Intensity Measurements at Normal Incidence, Appl. Opt., 1968, 7, 435-442, DOI: 10.1364/ao.7.000435.

22 S. Maeda, P. N. Schatz and G. Thyagarajan, Absolute Infrared Intensity Measurements in Thin Films .2. Solids Deposited on Halide Plates, J. Chem. Phys., 1963, 39, 34743481, DOI: 10.1063/1.1734216.

23 R. Nitsche and T. Fritz, Determination of model-free Kramers-Kronig consistent optical constants of thin absorbing films from just one spectral measurement: Application to organic semiconductors, Phys. Rev. B, 2004, 70, 195432, DOI: 10.1103/PhysRevB.70.195432. 
24 H. G. Yan, et al., Tunable infrared plasmonic devices using graphene/insulator stacks, Nat. Nanotechnol., 2012, 7, 330334, DOI: 10.1038/nnano.2012.59.

25 Y. Shen, et al., Optical investigation of reduced graphene oxide by spectroscopic ellipsometry and the band-gap tuning, Appl. Phys. Lett., 2011, 99, 141911, DOI: 10.1063/1.3646908.

26 M. Acik, et al., Unusual infrared-absorption mechanism in thermally reduced graphene oxide, Nat. Mater., 2010, 9, 840-845, DOI: $10.1038 /$ nmat2858.

27 S. Fraser, X. R. Zheng, L. Qiu, D. Li and B. H. Jia, Enhanced optical nonlinearities of hybrid graphene oxide films functionalized with gold nanoparticles, Appl. Phys. Lett., 2015, 107, 031112, DOI: 10.1063/1.4927387.

28 M. Beck, et al., Continuous wave operation of a mid-infrared semiconductor laser at room temperature, Science, 2002, 295, 301-305, DOI: 10.1126/science.1066408.

29 J. Rosenberg, R. V. Shenoi, T. E. Vandervelde, S. Krishna and O. Painter, A multispectral and polarization-selective surface-plasmon resonant midinfrared detector, Appl. Phys. Lett., 2009, 95, 161101, DOI: 10.1063/1.3244204.

30 I. Yamada, et al., Mid-infrared wire-grid polarizer with silicides, Opt. Lett., 2008, 33, 258-260, DOI: 10.1364/ ol.33.000258.

31 K. J. Lee, J. Giese, L. Ajayi, R. Magnusson and E. Johnson, Resonant grating polarizers made with silicon nitride titanium dioxide and silicon: Design fabrication and characterization, Opt. Express, 2014, 22, 9271-9281, DOI: 10.1364/ oe.22.009271.

32 I. Yamada, K. Fukumi, J. Nishii and M. Saito, Infrared wiregrid polarizer with Y2O3 ceramic substrate, Opt. Lett., 2010, 35, 3111-3113, DOI: 10.1364/ol.35.003111.

33 K. Shiraishi, S. Higuchi, K. Muraki and H. Yoda, Silver-film subwavelength gratings for polarizers in the terahertz and mid-infrared regions, Opt. Express, 2016, 24, 20177-20186, DOI: 10.1364/oe.24.020177.
34 Y. R. Fang, Y. Q. Ge, C. Wang and H. Zhang, Mid-Infrared Photonics Using 2D Materials: Status and Challenges, Laser Photonics Rev., 2020, 14, 1900098, DOI: 10.1002/ lpor.201900098.

35 S. H. Fan and J. D. Joannopoulos, Analysis of guided resonances in photonic crystal slabs, Phys. Rev. B, 2002, 65, 235112, DOI: 10.1103/PhysRevB.65.235112.

36 V. Lousse, et al., Angular and polarization properties of a photonic crystal slab mirror, Opt. Express, 2004, 12, 15751582, DOI: 10.1364/opex.12.001575.

37 P. G. Hermannsson, C. Vannahme, C. L. C. Smith and A. Kristensen, Absolute analytical prediction of photonic crystal guided mode resonance wavelengths, Appl. Phys. Lett., 2014, 105, 071103, DOI: 10.1063/1.4893664.

38 P. G. Hermannsson, et al., All-polymer photonic crystal slab sensor, Opt. Express, 2015, 23, 16529-16539, DOI: 10.1364/oe.23.016529.

39 J. Li, B. Jia, G. Zhou and M. Gu, Direction-dependent spontaneous emission from near-infrared quantum dots at the angular band edges of a three-dimensional photonic crystal, Appl. Phys. Lett., 2007, 91, 254101, DOI: 10.1063/ 1.2824388 .

40 J. Y. Ma, F. Zhu and C. H. Zhou, Wideband Polarizer in the Middle IR Wavelength Region Based on Guided Mode Resonance Effect, IEEE Photonics Technol. Lett., 2014, 26, 1364-1367, DOI: 10.1109/lpt.2014.2324578.

41 A. J. Berndt, et al., Poly(sulfur-random-(13-diisopropenylbenzene)) based mid-wavelength infrared polarizer: Optical property experimental and theoretical analysis, Polymer, 2019, 176, 118-126, DOI: 10.1016/j. polymer.2019.05.036.

42 D. J. Griffiths, Introduction to Electrodynamics, Cambridge University Press, 4th edn, 2017.

43 Thorlabs, https://www.thorlabs.com/newgrouppage9.cfm? objectgroup_id=1118. 